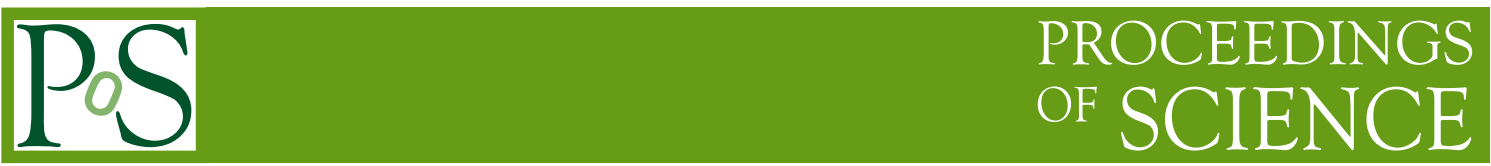

\title{
The Latest Tests of the SFXC Software Correlator
}

\section{Yurii Pidopryhora*}

Joint Institute for VLBI in Europe, the Netherlands

E-mail: pidopryhoraejive.nl

\section{Aard Keimpema}

Joint Institute for VLBI in Europe, the Netherlands

E-mail: keimpema@jive.nl

\section{Mark Kettenis}

Joint Institute for VLBI in Europe, the Netherlands

E-mail: kettenis@jive.nl

Recently a number of improvements were made to the SFXC software correlator developed within the EXPReS FABRIC research activity and the SCARIe project. It has been tested by processing data from an actual VLBI experiment. We are presenting a comparison of accuracy with the EVN MkIV hardware correlator and the DiFX software correlator, which proves to be an excellent match.

Science and Technology of Long Baseline Real-Time Interferometry:

The 8th International e-VLBI Workshop, EXPReSO9

June 22 - 262009

Madrid, Spain

${ }^{*}$ Speaker. 

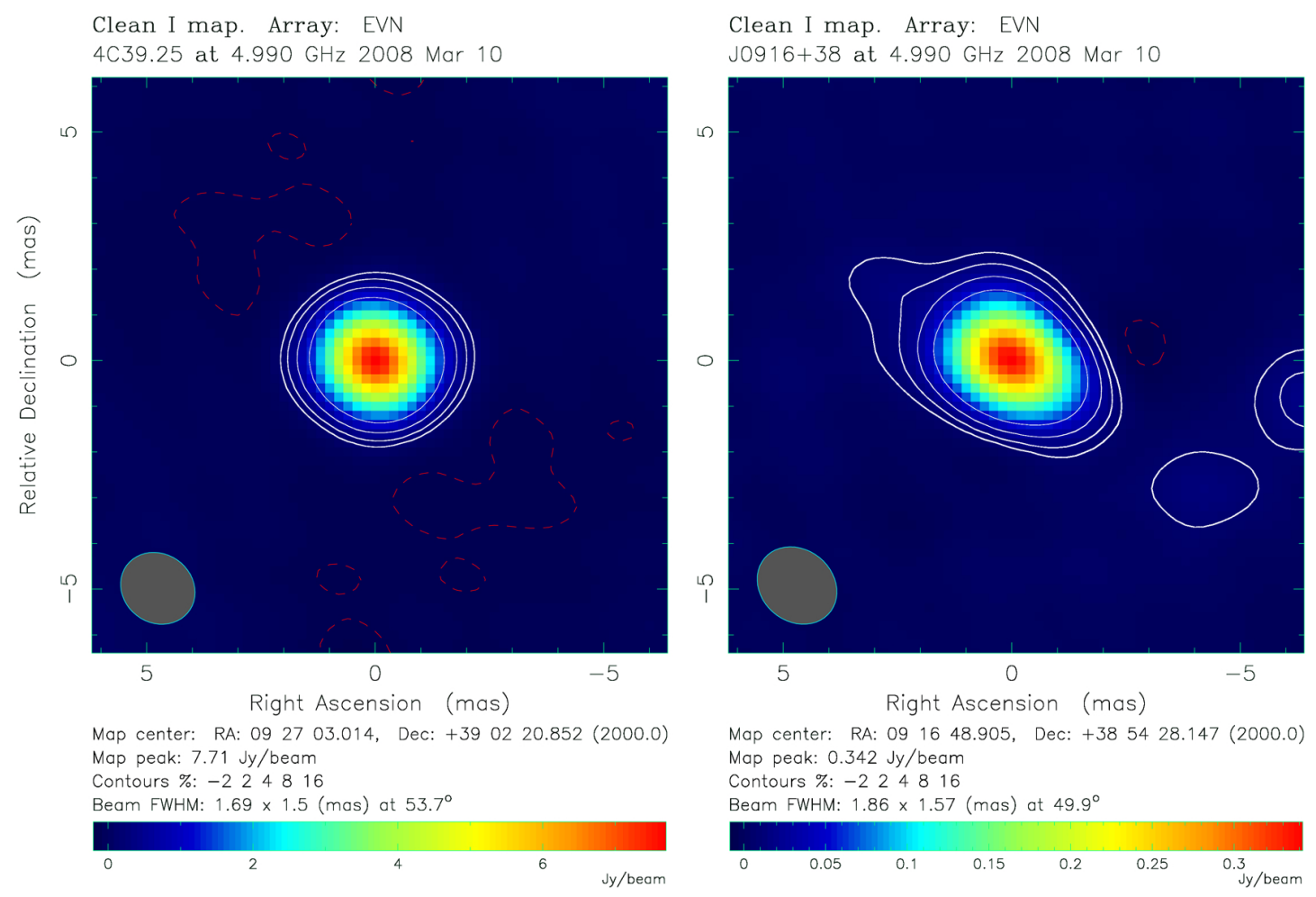

Figure 1: The images of two VLBI calibrator sources, 4C39.25 (left) and J0916+38 (right) produced with the data correlated with the SFXC software correlator during this testing run (all frequency subbands and polarizations averaged).

The SFXC software correlator has been developed at JIVE as a part of the FABRIC (Future Arrays of Broadband Radio-telescopes on Internet Computing) research activity of EXPReS (Express Production Real-time e-VLBI Service) project and the SCARIe (Software Correlator Architecture Research and Implementation for e-VLBI) project [1]. Recently we have used $\approx 2 \mathrm{hrs}$ of data from a C-band EVN network monitoring experiment (N08C1) for an extensive test of the correlator. In this segment of the experiment two sources, 4C39.25 and J0916+38, both standard VLBI calibrators $[2,3]$ were observed in four $8 \mathrm{MHz}$-wide subbands in both RCP and LCP. The resulting images are shown in Figure 1. The correlation was performed using 32 frequency points and a $2 \mathrm{~s}$ integration interval. The same data were correlated with the EVN MkIV hardware correlator [4] using the same correlation parameters. Also some few-minute-long portions of the data were correlated with the DiFX software correlator [5]. The raw correlation output of all the three correlators was then compared against each other. In particular, fringe amplitudes and phases were compared and their noise levels were analyzed.

Figure 2 shows a typical example of phase comparison between the SFXC and EVN MkIV correlators for the whole data sample. Data corresponding to different sources are plotted separately since, at this frequency, 4C39.25 is about 20 times brighter than J0916+38 and thus the noise levels are significantly different. An example of comparison of all three correlators over a 3-minute 

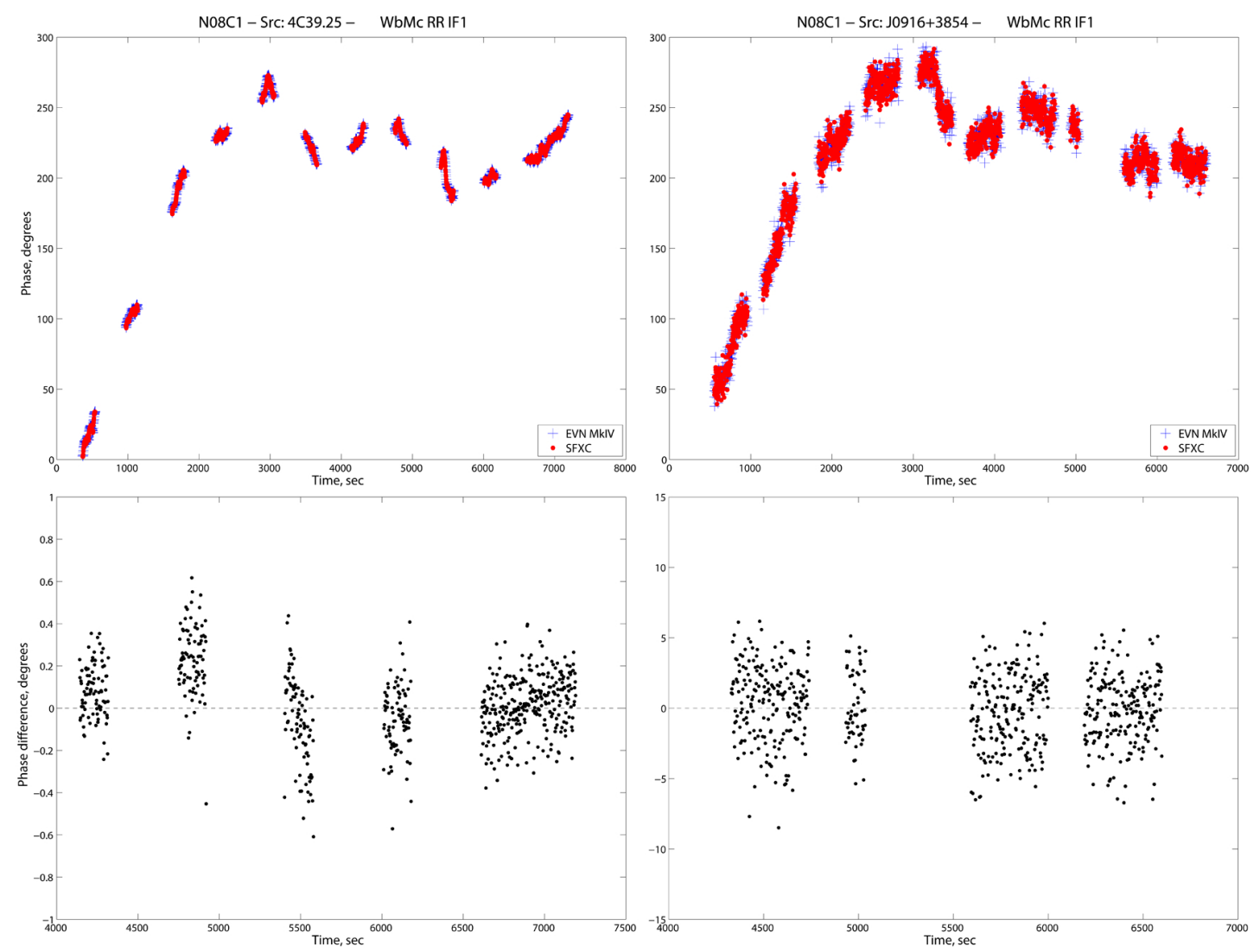

Figure 2: A typical example of phase comparison between SFXC software (red dots) and EVN MkIV (blue crosses) hardware correlators for a particular baseline (Westerbork-Medicina), polarization (RR) and frequency subband $(4.97449 \mathrm{GHz})$. The upper panels show the phase of both correlators plotted for the whole length of the test data sample $(\approx 2 \mathrm{hrs})$ The lower panels show the difference between the SFXC and EVN MkIV phases over a part of that time. The two left panels are for 4C39.25 and the right ones are for J0916+38. They are plotted separately because of the different noise levels (note the different $y$-scales of the left and right phase difference plots).

interval is shown in Figure 3. The right panel of that figure shows the corresponding noise analysis graph. For this purpose we have employed the Allan standard deviation $\sigma$, a function of time interval $\tau$, which is calculated as [6]:

$$
\sigma^{2}(\tau)=\frac{\left\langle[\varphi(t+2 \tau)-2 \varphi(t+\tau)+\varphi(t)]^{2}\right\rangle}{8 \pi^{2} v^{2} \tau^{2}}
$$

where $\varphi$ is the fringe phase, $v$ is the observing frequency and angular brackets denote sample averaging over all $t$.

In the course of this testing hundreds of samples for different baselines, frequency bands and polarizations processed with SFXC and the two other correlators were compared and all of them match well. Noise levels of particular samples show slight differences, but overall S/N ratio of all the three correlators is the same. Thus we can conclude that the SFXC correlator checks well 

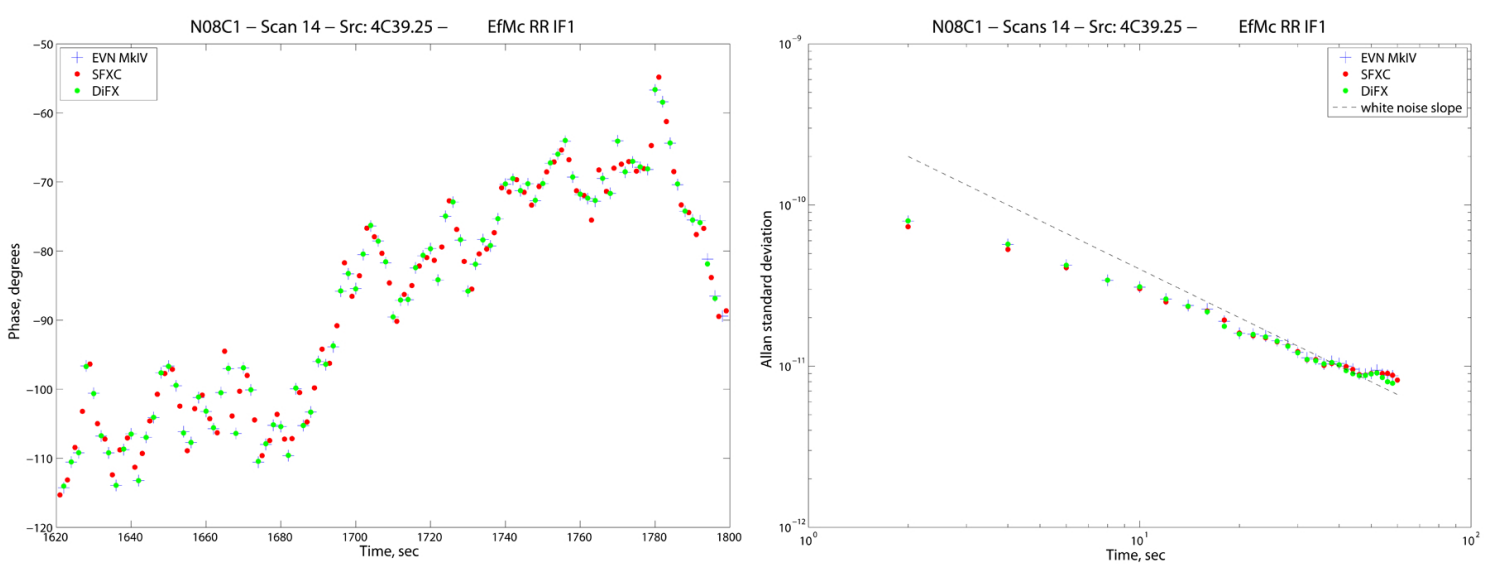

Figure 3: Left panel: a typical example of phase comparison between the three correlators: SFXC (red dots), DiFX (green dots), and EVN MkIV (blue crosses), taken over 3 minutes of the test data. Baseline for this sample is Effelsberg-Medicina, polarization is RR and frequency is $4.97449 \mathrm{GHz}$. Note that although integration interval is the same, $2 \mathrm{~s}$, SFXC data points are for odd seconds and MkIV/DiFX are for even ones. Right panel: Allan standard deviation for the same sample. The dashed line shows the slope of the ideal white noise.

against both EVN Mark IV hardware and DiFX software correlators and hence it is fully usable for processing astronomical data.

The authors acknowledge funding within FABRIC/EXPReS and SCARIe projects, supported by the Nederlandse Organisatie voor Wetenschappelijk Onderzoek (NWO). EXPReS is an Integrated Infrastructure Initiative (I3), funded under the European Commission's Sixth Framework Programme (FP6), contract number 026642, from March 2006 through February 2009.

\section{References}

[1] N. Kruithof, e-VLBI Using a Software Correlator, in book Grid Enabled Remote Instrumentation, series Signals and Communication Technology, eds. F. Davoli, N. Meyer, R. Pugliese, and S. Zappatore, Springer US (2009) 537.

[2] C. Ma, E.F. Arias, T.M. Eubanks, A.L. Fey, A.-M. Gontier, C.S. Jacobs, O.J. Sovers, B.A. Archinal, and P. Charlot, The International Celestial Reference Frame as Realized by Very Long Baseline Interferometry, Astron. J 116 (1998) 516.

[3] A.J. Beasley, D. Gordon, A.B. Peck, L. Petrov, D.S. MacMillan, E.B. Fomalont, and C. Ma, The VLBA Calibrator Survey - VCS1, Astroph. J. Sup 141 (2002) 13.

[4] R.T. Schilizzi, W. Aldrich, B. Anderson, A. Bos, R.M. Campbell, J. Canaris, R. Cappallo, J.L. Casse, A. Cattani, J. Goodman, H.J. van Langevelde, A. Maccafferri, R. Millenaar, R.G. Noble, F. Olnon, S.M. Parsley, C. Phillips, S.V. Pogrebenko, D. Smythe, A. Szomoru, H. Verkouter, and A.R. Whitney, The EVN-MarkIV VLBI Data Processor, Experimental Astronomy 12 (2001) 49.

[5] A.T. Deller, S.J. Tingay, M. Bailes, and C. West, DiFX: A Software Correlator for Very Long Baseline Interferometry Using Multiprocessor Computing Environments, PASP 119 (2007) 318.

[6] A.R. Thompson, J.M. Moran, and G.W. Swenson Jr., Interferometry and Synthesis in Radio Astronomy, Wiley-Interscience, New York 2001. 